\section{HEDGEHOG SIGNALING DRIVES EPITHELIAL-TO- MESENCHYMAL TRANSITION, IMMUNE EVASION, AND ANTI-PD-1 RESISTANCE THROUGH COORDINATED UPREGULATION OF WNT LIGANDS AND PGE2 SYNTHESIS}

${ }^{1}$ Nicholas DeVito*, ${ }^{2}$ Michael Sturdivant, ${ }^{1}$ Balamayooran Theivanthiran, ${ }^{1} Y$-Van Nguyen, ${ }^{1}$ Michael Plebanek, 'Brent Hanks. 'Duke University Medical Center, Durham, NC, USA; ${ }^{2}$ University of North Carolina, Chapel HIII, NC, USA

Background Immunotherapy resistance has been correlated with epithelial-to-mesenchymal transition (EMT), ${ }^{1}{ }^{2}$ however our understanding of tumor-intrinsic mechanisms driving this immune evasive phenotype is lacking. We have previously shown that Wnt ligands are upregulated in anti-PD-1 resistant melanomas, ${ }^{3}$ and postulated that upstream transcriptional regulation of select EMT pathways may underpin these findings. The hedgehog signaling $(\mathrm{HH})$ transcription factor Gli2 promotes EMT.

Methods Gli2 was constitutively activated (Gli2 ${ }^{\mathrm{CA}}$ ) in a $\mathrm{BRAF}^{\mathrm{V} 600 \mathrm{E}} \mathrm{PTEN}^{-/}$murine cell line via an $\mathrm{N}$-terminal truncating mutation and silenced using CRISPR-Cas9. Multi-parameter flow cytometry and RNAseq was utilized to evaluate the impact of Gli2 on the tumor immune microenvironment. Anti-PD-1 resistance studies were performed in Gli2 $2^{\mathrm{CA}}$ and control tumors. Bioinformatics studies were conducted using the melanoma TCGA and Hugo et al databases. ${ }^{2}$

Results We found upregulation of Gli2 targets in patients with anti-PD-1-refractory metastatic melanoma as well as in an autochthonous $\mathrm{BRAF}^{\mathrm{V} 600 \mathrm{E}} \mathrm{PTEN}^{-/}$melanoma model after escape from anti-PD-1. RNAseq and Western blot studies demonstrated Gli2 ${ }^{\mathrm{CA}}$ to promote EMT and Wnt ligand production in addition to upregulated COX2 in $\mathrm{BRAF}^{\mathrm{V} 600 \mathrm{E}} \mathrm{PTEN}^{-/}$melanoma. This finding was reversed by genetic ablation and pharmacologic inhibition of Gli2, implicating a previously undescribed role for Gli2 in modulating COX2. These data were consistent with a notable correlation between a Gli2 signature and a prostaglandin synthesis signature in human melanoma TCGA database. Flow cytometry analysis showed exclusion of cytolytic $\mathrm{T}$ and NK cells, a shift from cDC1s to cDC2s, and enhanced MDSC recruitment in Gli2 ${ }^{\mathrm{CA}}$ tumors. Consistent with these findings, whole tumor RNAseq of Gli2CA tumors demonstrated a decrease in $\mathrm{Cd} 3 e$, Prf1, and Xcr1 with a concomitant increase in Cxcl1, Cxcl2, Ccl2, Ptgs2, and Arg1 relative to control tumors. RNAseq of FACSsorted DCs from Gli2 ${ }^{\mathrm{CA}}$ tumors demonstrated a loss of cDC1-associated genes including Xcr1, Wdfy4, and Clec9a compared to DCs derived from control tumors. In-line with our previous results showing that Wnt5a promotes MDSC recruitment in a Yap-dependent manner, ${ }^{4}$ we found that Yap inhibition or Wnt5a deletion in the $\mathrm{BRAF}^{\mathrm{V} 600 \mathrm{E}} \mathrm{PTEN}^{-/-} \mathrm{Gli} 2{ }^{\mathrm{CA}}$ cell line diminished MDSC-recruiting chemokines. Further consistent with these findings, Gli2 ${ }^{\mathrm{CA}}$ tumors resist anti-PD-1 antibody therapy.

Conclusions Our data demonstrates that the $\mathrm{HH}$ transcription factor Gli2 drives the development of a tolerogenic tumor microenvironment unfavorable to anti-PD-1 immunotherapy by coordinating the upregulation of Wnt ligand expression and prostaglandin synthesis (figure 1). We propose that $\mathrm{HH}$ gene signatures are worthy of further study as a guide for selecting Wnt ligand and prostaglandin inhibitors in future immunotherapy studies.

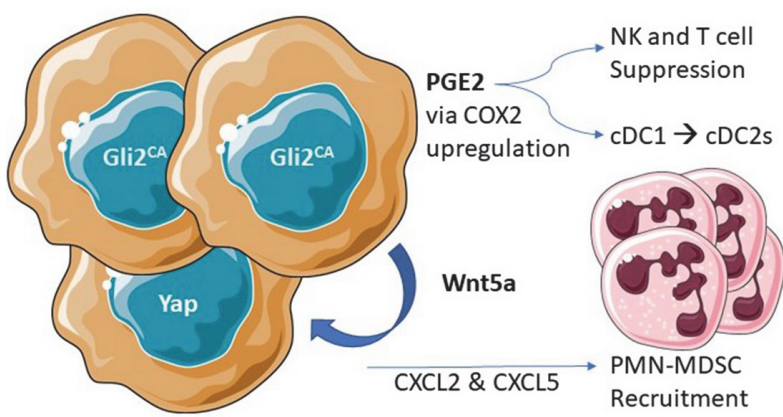

Abstract 923 Figure $1 \mathrm{Gli2}$ in tumors promotes Wnt and prostaglandin signaling, generating an immunosuppressive microenvironment

Acknowledgements The authors would like to acknowledge the Duke Cancer Institute Flow Cytometry Core.

\section{REFERENCES}

1. Bagaev $A$, et al. Conserved pan-cancer microenvironment subtypes predict response to immunotherapy. Cancer Cell 2021.

2. Hugo W, et al. Genomic and transcriptomic features of response to anti-PD-1 therapy in metastatic melanoma. Cell 2016;165(1):35-44.

3. DeVito NC, et al. Pharmacological Wnt ligand inhibition overcomes key tumor mediated resistance pathways to anti-PD-1 immunotherapy. Cell Rep 2021;35 (5): 109071

4. Theivanthiran B, et al. A tumor-intrinsic PD-L1/NLRP3 inflammasome signaling pathway drives resistance to anti-PD-1 immunotherapy. J Clin Invest 2020;130 (5):2570-2586.

http://dx.doi.org/10.1136/jitc-2021-SITC2021.923 